\title{
ALGEBRAIC VECTOR BUNDLES OVER THE PRODUCT OF AN AFFINE CURVE AND THE AFFINE LINE
}

\section{S. SESHADRI}

Let $k$ be an algebraically closed field and $A$ the affine algebra of a nonsingular irreducible affine curve defined over $k$. Then $A[X]$, the algebra of polynomials over $A$, is the affine algebra of the product variety $C \times k^{1}$. Let $P$ be a projective module of finite type over $A[X]$. The module $P$ corresponds to an algebraic vector bundle over $C \times k^{1}[4, \S 4$, Chapter II $]$. The purpose of this note is to prove the following

Theorem. There exists a projective module $\Lambda$ over $A$ of finite type, such that

$$
P=\Lambda \otimes_{A} A[X]
$$

(i.e. every algebraic vector bundle over $C \times k^{1}$ can be obtained as the inverse image of an algebraic vector bundle over $C$, by the projection mapping $\left.C \times k^{1} \rightarrow k^{1}\right)$.

This result generalizes the one proved in [6] that every algebraic vector bundle over $k^{2}$ is trivial. The method of proof is also similar to $[6]$.

Let $B$ be a normal (integrally closed) noetherian ring. Then a divisor $D$ in $B[3, \S 2$, Chapter III $]$ is a finite linear combination with integral coefficients of prime ideals of height 1 (minimal prime ideals). Let $S$ be a multiplicatively closed subset in $B$, not containing 0 and containing 1 , and $B S^{-1}$ the ring of classes of fractions $b / s, b \in B, s \in S$. Then for a divisor $D=\sum_{i \in I} a_{i} p_{i}$ in $B$ ( $p_{i}$ prime, $a_{i}$ integer) we define a divisor $D S^{-1}$ in $B S^{-1}$ by

$$
D S^{-1}=\sum_{j \in J} a_{j}\left(p_{j} S^{-1}\right),
$$

$J$ being the subset of $I$ containing those indices for which $p_{i}$ does not intersect $S$.

Proposition 1. Every divisor $D^{\prime}$ in $B S^{-1}$ can be put in the form $D S^{-1}$ where $D$ is a divisor in $B$.

We have only to prove that every prime ideal $p^{\prime}$ of height 1 in $B S^{-1}$ can be expressed in the form $p S^{-1}$ where $p$ is a prime ideal of

Received by the editors November 24, 1958. 
height 1 in $B$. This is an immediate consequence of $[2$, Proposition 1 , Exposé 1].

Proposition 2. Let $B$ further satisfy the condition, that for every maximal ideal $m$, the localized ring $B_{m}$ (i.e. $B_{m}=B S^{-1}$ with $S=B-m$ ) is factorial. Then for every projective module $N$ over $B S^{-1}$ of rank 1 , there exists a projective module $M$ over $B$ of rank 1 , such that $N$ is isomorphic to $M \otimes_{B} B S^{-1}$.

$N$ is isomorphic to an inversible ideal (cf. $[1, \S 3$, Chapter VII ]) in $B S^{-1}$. Let $\mathfrak{A}$ be such an ideal. It is easy to verify that $\left(B S^{-1}:\left(B S^{-1}: \mathfrak{A}\right)\right)$ $=\mathfrak{A}$. Therefore $\mathfrak{A}$ is the ideal of a divisor $D^{\prime}$ in $B S^{-1}$ (cf. [3, Corollary, Theorem 4, §2, Chapter III]). By Proposition 1, $D^{\prime}$ can be "lifted" to a divisor $D$ in $B$. The ideal $I(D)$ of the divisor $D$ is "locally" principal because of the hypothesis that $B_{m}$ is factorial for every maximal ideal $m$ (cf. [3, Theorem 1, $\S 3$, Chapter III]). Therefore $I(D)$ is an inversible ideal and corresponds to a projective module $M$ over $B$ of rank 1 (cf. [5, Proposition 3]). It is easily verified that $N$ is isomorphic to $M \otimes_{B} B S^{-1}$.

Corollary. Proposition 2 is valid when $B$ is the affine algebra of a nonsingular irreducible affine variety.

For, in this case, it is well-known that every $B_{m}$ is factorial.

Let now $A$ be the affine algebra of an irreducible nonsingular affine curve $C$ defined over an algebraically closed field $k$ and $P$ a projective module of finite type over $B=A[X]$. Then $P$ corresponds to an algebraic vector bundle over $C \times k^{1}$. In order to prove the theorem it can be assumed that $P$ is of rank $\leqq 2$, since by a theorem of Serre [5, Theorem 1], $P=L_{1}+P_{1}$, where $L_{1}$ is free and $P_{1}$ is of rank $\leqq 2$. Let $S$ be $k[X]^{*}$, the set of all nonzero polynomials over $k$. When $P$ is of rank 1 , the theorem stated is well-known. Therefore, we suppose hereafter that $P$ is of rank 2. Consider $P S^{-1}$. This is a projective module over $B S^{-1}$ (the affine algebra of the curve $C$ extended to $K=k(X)$ ) of rank 2. Then $B S^{-1}$ is a Dedekind ring, so that $P \otimes_{k[X]} k(X)=P S^{-1}=N_{1}+N_{2}$, where $N_{1}$ is a free module over $B S^{-1}$ of rank 1 and $N_{2}$ a projective module over $B S^{-1}$ of rank 1 (cf. [5, Proposition 7]). We can choose elements $y_{1}, z_{1}, \cdots, z_{p}$ of $P$ and $a \in k[X]^{*}$ such that $y_{1} \otimes 1 / a$ generates $N_{1}$ and $z_{1} \otimes 1 / a, \cdots, z_{p} \otimes 1 / a$ generate $N_{2}$. Because of Proposition 2 it can be supposed that $z_{1}, \cdots, z_{p}$ generate a projective submodule $M_{2}$ of $P$ of rank 1 . Let $M_{1}$ be the free submodule of $P$ of rank 1 , generated by $y_{1}$. Then $L=M_{1}+M_{2}$ is a submodule of $P$ satisfying the following conditions:

(i) $L$ is a direct sum of projective modules of rank 1 ; 
(ii) $a P \subset L \subset P, a \in k[X]^{*}$.

Further it can be supposed that

(iii) $(L: P)=(a)$, and

(iv) If $L_{1}$ is a submodule of $P$ satisfying (i) and (iii) in place of $L$ and $L \subset L_{1} \subset P$, then it should follow that $L=L_{1}$.

If " $a$ " were a unit, the required theorem is proved, otherwise $\exists$ a prime $p(p \neq 1)$ of $k[X]$ which divides $a$. Let $a=a_{1} p$. Because of the property $L: P=(a)$ on account of (iii), $\exists$ an element $x \in P$ such that $a_{1} x \in L$ and $p a_{1} x \in L$. This shows that $L \cap p P \neq p L$. Now consider the homomorphism

$$
\text { II: } L / p L \rightarrow P / p P
$$

induced by the inclusion $L \subset P$. Then $P$ gives rise to the following exact sequence

$$
0 \rightarrow \operatorname{Ker} \Pi \rightarrow L / p L \rightarrow \operatorname{Im} \Pi \rightarrow 0
$$

of modules over the ring $(k[X] /(p)) \otimes_{k[X]} A[X]$ which is canonically isomorphic to $A$, since $k$ is algebraically closed. By means of this isomorphism, we consider the modules in the exact sequence (1) as modules over $A$. Then Im II is a projective module over $A$, since it is a submodule of $P / p P$ which is a projective module over the Dedekind ring $A$. Therefore (1) splits and we have

$$
L / p L=\operatorname{Ker} \Pi+\operatorname{Im} \Pi \text {. }
$$

Since $L$ is a direct sum of projective modules of rank 1 and the theorem stated is known to be true for projective modules of rank $1, L$ is isomorphic to $(L / p L) \otimes_{A} A[X]$. Therefore the decomposition (2) can be "lifted" to the following decomposition as modules over $A[X]$ :

$$
L=M+N \text { with } M / p M=\operatorname{Ker} \Pi, \quad N / p N=\operatorname{Im} \Pi \text {. }
$$

Since $L \cap p P \neq p L$, Ker $\Pi \neq 0$. Therefore, since $L / p L$ is of rank 2, either Ker $\Pi$ is of rank 2 and $I m \Pi$ is 0 , or Ker $\Pi$ and $I m \Pi$ are both of rank 1 respectively. In the first case, $\exists$ a submodule $L_{1}$ of $P$ such that $p L_{1}=L$. Then $L_{1}$ is obviously a direct sum of projective modules of rank 1 and

$$
a_{1} P \subset L_{1} \subset P, \quad a_{1} p=a .
$$

In the second case $\exists$ a projective submodule $M_{1}$ of $P$ (of rank 1) such that $p M_{1}=M$. It is trivial to verify that $M_{1}$ contains $M$ strictly. Let $L_{1}=M_{1}+N$. Then $L_{1}$ is a direct sum of projective modules of rank 1 and contains $L$ strictly, so that by (iv)

$$
b P \subset L_{1} \subset P \text {. }
$$


$b$ being proper divisor of $a$.

Thus, in any case when $a$ is not a unit, we obtain $\theta P \subset L_{1} \subset P$, $\theta$ being a proper divisor of $a$ and $L_{1}$ being a direct sum of projective modules of rank 1 . By repeating the process, we find that $P$ is itself a direct sum of modules of rank 1 and as the theorem is known to be true for projective modules of rank 1 , it is proved.

Finally, the author would like to express his thanks to Professor J. P. Serre for the many kind suggestions he received from him.

\section{REFERENCES}

1. H. Cartan and S. Eilenberg, Homological algebra, Princeton, 1956.

2. H. Cartan et C. Chevalley, Géométrie algébrique, Séminaire E.N.S., 1955-1956.

3. P. Samuel, Commutative algebra, Notes by D. Hertzig, Cornell University, 1953.

4. J. P. Serre, Faisceaux algébriques cohêrents, Ann. of Math. vol. 61 (1955) pp. 197-278.

5. - Modules projectifs et espaces fibrés a fibre vectorielle, Séminaire P. Dubriel, 1957-1958.

6. C. S. Seshadri, Triviality of vector bundles over the affine space $K^{2}$, Proc. Nat. Acad. Sci. U.S.A. vol. 44 (1958) pp. 456-458.

Tata Institute of Fundamental Research, Bombay and

Centre National de la Recherche Scientifique, Paris 